\title{
Ostial stenosis of the left main coronary artery as the result of the previous percutaneous coronary intervention on the left coronary artery
}

\author{
Grzegorz Mencel, Jacek Kowalczyk, Marcin Swierad, Andrzej Swiatkowski, Grzegorz Honisz, Zbigniew Kalarus, \\ Beata Sredniawa
}

Department of Cardiology, Congenital Heart Diseases and Electrotherapy, Medical University of Silesia, Silesian Center for Heart Diseases, Zabrze, Poland

Postep Kardiol Inter 2013; 9, 3 (33): 317-320

DOI: $10.5114 /$ pwki.2013.37521

\begin{abstract}
A 76-year-old patient was admitted to hospital due to recurrent chest pain. Angiography revealed multivessel coronary artery disease with significant stenosis in medial and distal parts of the left main coronary artery (LMCA). Two drug-eluting stents (DES) were implanted from the medial part of the LMCA to the proximal part of the left anterior descending (LAD) artery. An excellent angiographic result was achieved. After 20 days the patient returned to our clinic because of acute coronary syndrome (ACS). Angiography showed $80 \%$ stenosis in the ostial LMCA. The lesion was stented with DES, obtaining a good angiographic result.
\end{abstract}

Key words: left main coronary artery, restenosis.

\section{Introduction}

Significant left main coronary artery disease $(>50 \%$ diameter stenosis) is found in 4-10\% patients who undergo coronary angiography, and is associated with worse prognosis in the follow-up [1-3]. In accordance with the present guidelines on myocardial revascularization of the European Society of Cardiology (ESC), patients with left main coronary artery (LMCA) stenosis are appropriate candidates for coronary artery bypass grafting (CABG) as well as percutaneous coronary intervention (PCI) [4]. The key factor which determines the treatment strategy is the result presented in the risk stratification scale (SYNTAX score) as well as localization of the lesion in the left main. Another essential factor is the coexistence of 1-, 2- or 3-vessel disease [4]. Another issue is acute coronary syndrome (ACS) and/or accompanying cardiogenic shock, due to LMCA stenosis requiring immediate $\mathrm{PCl}$.

\section{Case report}

A 76-year-old man of Caucasian origin with a history of non-ST-elevation myocardial infarction treated with a conservative strategy 6 months before was admitted to hos- pital due to recurrent chest pain for 7 days. The patient's concomitant diseases were heart failure with reduced ejection fraction (EF 38\%), arterial hypertension, dyslipidemia and chronic obstructive pulmonary disease. On admission the blood pressure was 130/60 mm Hg. An electrocardiogram (ECG) demonstrated ST segment depression by $1 \mathrm{~mm}$ in leads V5-V6 and a negative T wave in lead III. Biochemical investigations revealed an insignificant increase of cardiac troponins without its evolution.

The coronary angiography with intravascular ultrasound (IVUS) showed $80 \%$ obstruction in the medial part of the LMCA, $40 \%$ obstruction in the distal part of the LMCA and $90 \%$ stenosis in the proximal part (segment 6) of the left anterior descending (LAD) artery (Figure 1). Moreover, chronic total occlusion of the right coronary artery (RCA) and $90 \%$ stenosis of the obtuse marginal branch with diameter $<2 \mathrm{~mm}$ were revealed. Syntax Score for this patent was 27. A "heart team" was assembled and due to concomitant diseases and the high risk of the operation, the "heart team" qualified the patient for $\mathrm{PCl}$.

After balloon angioplasty (Falcon forte $3.0 \mathrm{~mm} \times 20 \mathrm{~mm}$ ), two DES (everolimus-eluting stents) were implanted. The first one (Xience $3.5 \mathrm{~mm} \times 33 \mathrm{~mm}$ ) was implanted into the

Corresponding author:

Grzegorz Mencel MD, Department of Cardiology, Silesian Center for Heart Diseases, 9 Curie-Sklodowskiej St, 41-800 Zabrze, Poland, tel.: +48 3227134 14, fax: +48 3237337 92, e-mail: grzesiekmen@gmail.com

Received: 30.04.2013, accepted: 28.06.2013. 


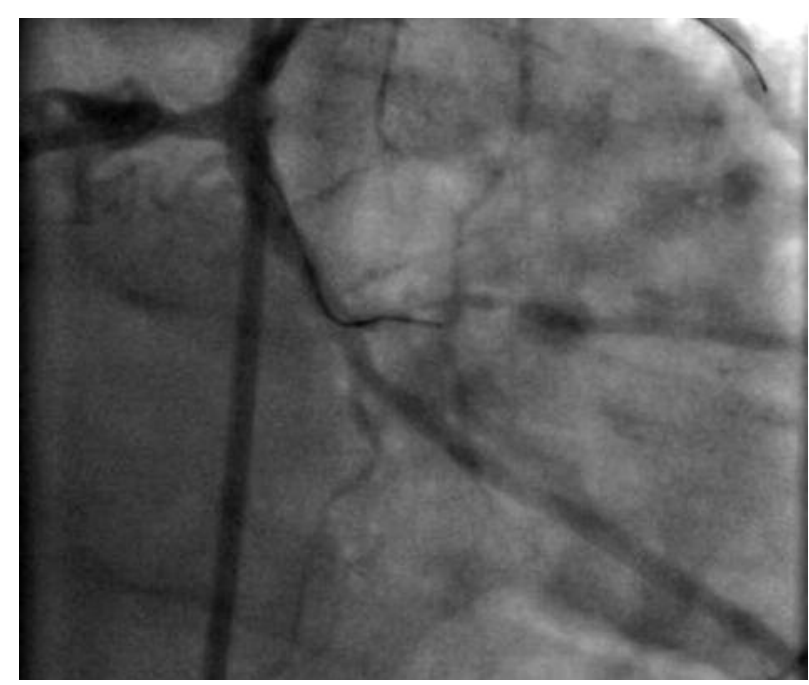

Fig. 1. Left coronary artery angiogram. LMCA at the beginning of the first procedure

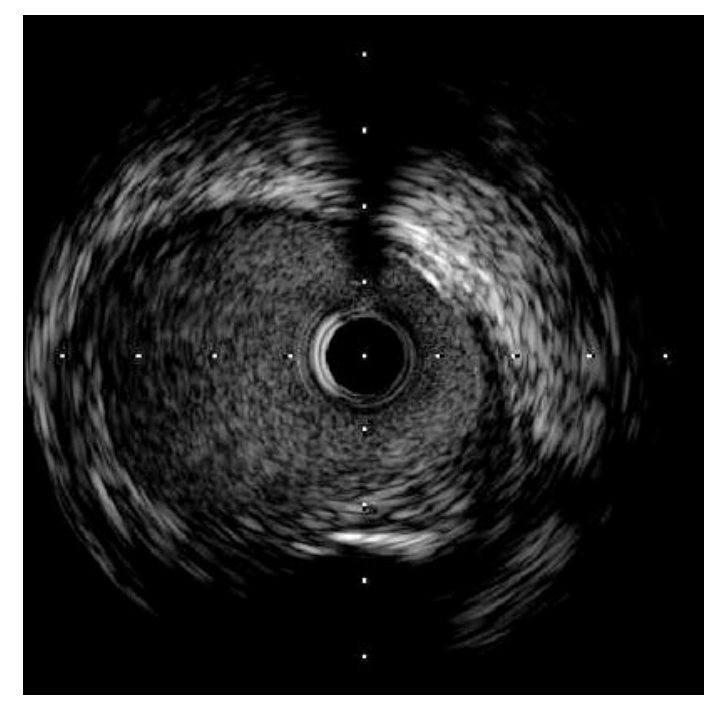

Fig. 3. IVUS of LMCA after the first procedure

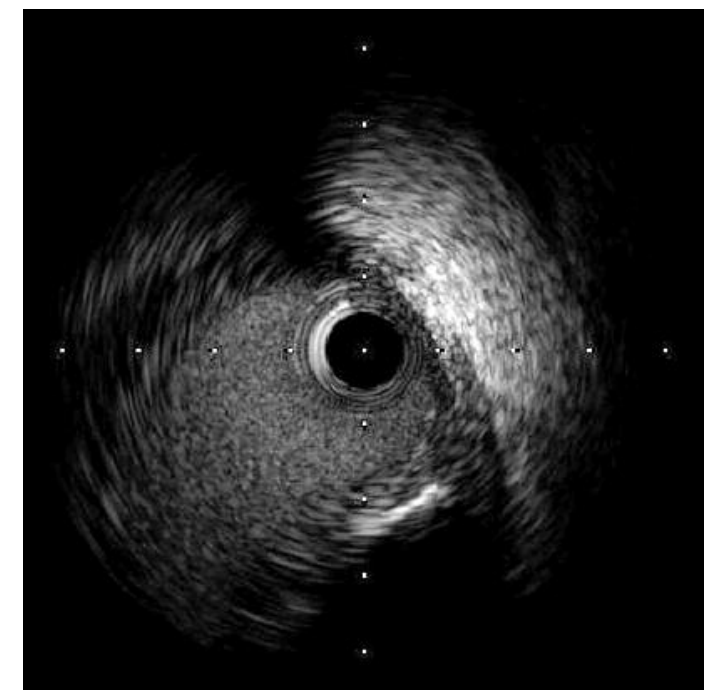

Fig. 5. IVUS of LMCA before the second procedure

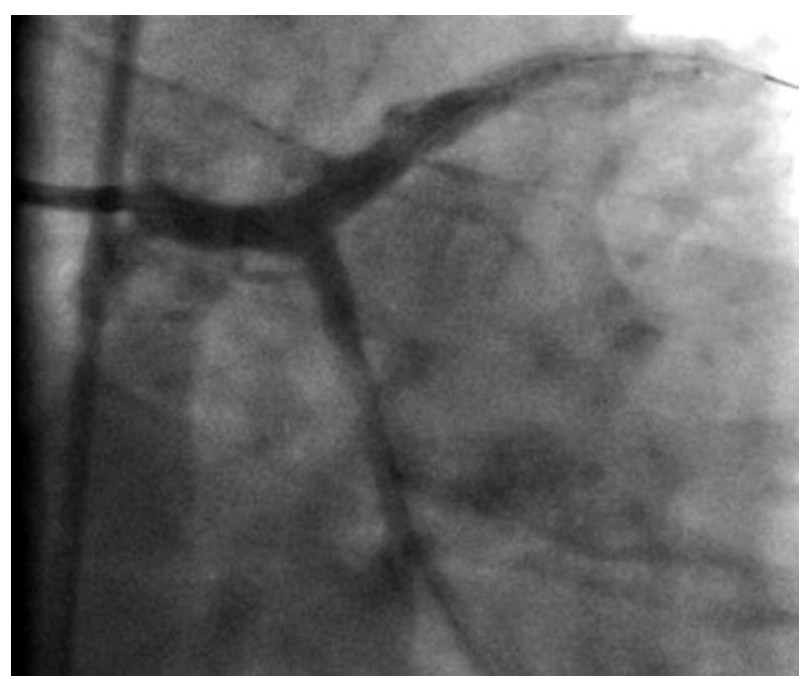

Fig. 2. Left coronary artery angiogram. LMCA final result of the first procedure

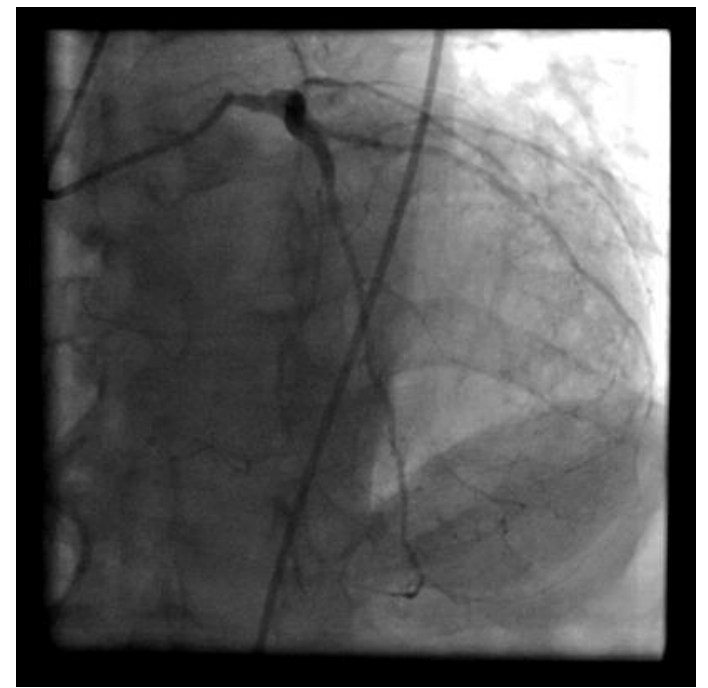

Fig. 4. Left coronary artery angiogram. LMCA before the second procedure

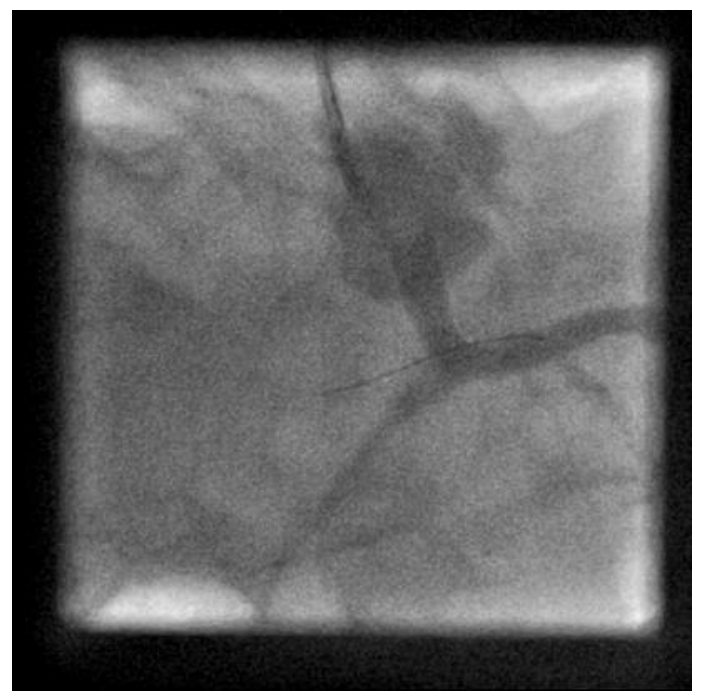

Fig. 6. Left coronary artery angiogram. LMCA after the second procedure 
proximal LAD. The second DES (Xience $4.0 \mathrm{~mm} \times 23 \mathrm{~mm}$ ) was deployed from the mid portion of the LMCA to the proximal part of the $6^{\text {th }}$ segment of the LAD, overlapping the first stent. Post-dilatation was performed using a kissing balloon technique (LMCA-LAD-CX). The final angiographic result was proper, which was confirmed in the control IVUS (Figures 2 and 3). After a 2-day rehabilitation, the patient was discharged from the hospital, with a double antiplatelet therapy recommendation (clopidogrel $75 \mathrm{mg} /$ day + aspirin $75 \mathrm{mg}$ /day). After 20 days the patient was admitted to our hospital again because of ACS. On admission the troponin level was elevated $(0.026 ; 0.079)$. ECG was comparable with the record from the previous hospitalization. The coronary angiography revealed $80 \%$ ostial stenosis of the LMCA, which was confirmed in IVUS (Figures 4 and 5). There was no restenosis in the previously implanted stents in the LMCA and LAD. Direct stenting was performed with a DES eluting Amphilimus $4.5 \mathrm{~mm} \times 12 \mathrm{~mm}$, which was deployed from the ostial to the medial section of the LMCA, overlapping the previously implanted stent to the LMCA-LAD. A very good final result was achieved, which was confirmed in IVUS (Figures 6 and 7). The further hospitalization continued without any complications. Routine control angiography was planned after three months.

\section{Discussion}

The present case is an example of ostial left main stenosis whose pathogenesis remains unclear. The previous procedures such as multiple LMCA intubation of a diagnostic catheter (JL 6 F), the use of a guiding catheter (launcher 6 F J 4.0, launcher 6 F EBU 4.0) and the movement of the IVUS, balloons and stents through the LMCA probably caused damage to the endothelial cells. latrogenic LMCA dissection as a complication of $\mathrm{PCl}$ had already been reported in the literature [5-8]. In most cases these were acute dissections caused by invasive procedures. There are no data concerning LMCA perturbation caused by stenting other coronary arteries and their possible consequences. The direct cause of the situation seems to be the dysfunction of endothelial cells whose pathogenesis might resemble the one in atherosclerosis. The main function of the endothelium is to sustain optimal blood flow through the vessel. It also prevents platelet adhesion as well as the formation of clots. Dysfunction of the endothelium leads to certain changes which subsequently activate procoagulant mechanisms whose key element is tissue factor (TF) induction [9]. The balance between vasodilators (prostacyclin (PGI2), nitric oxide (NO)) and vasoconstrictors (endothelin-1 (ET-1), thromboxane A2) is disturbed, which causes platelet and leukocyte adhesion to the endothelium cell surface $[9,10]$. The result of the above-mentioned mechanism was most likely LMCA ostial stenosis in this particular case. Due to ACS and the location of the lesion, $\mathrm{PCl}$ was performed with an optimal effect, which appeared to be the best solution for the patient.

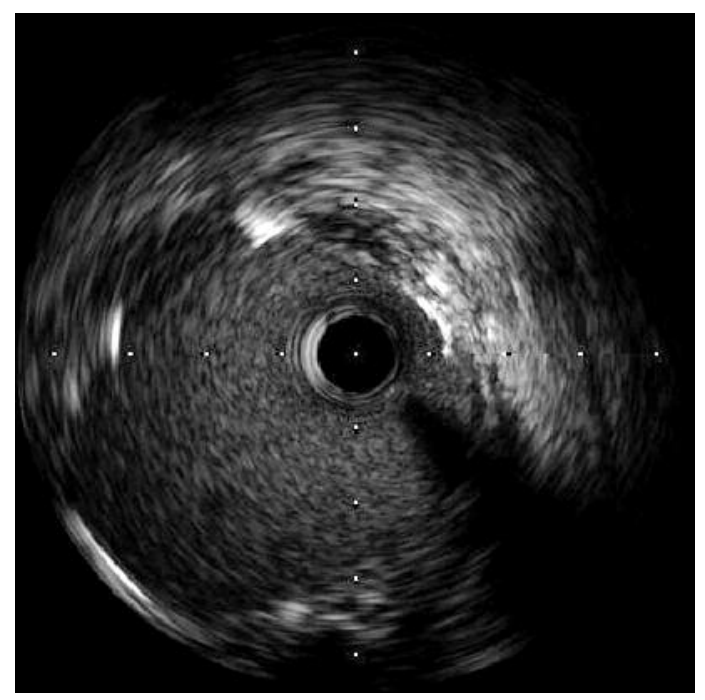

Fig. 7. IVUS of LMCA after the second procedure

The LMCA ostial stenosis may be the consequence of the previous intervention. Yet the functioning of this mechanism remains unknown. The lack of any similar cases of this kind in the literature may prove that the risk of changes caused by the procedures is highly unlikely. However, the risk seems to rise together with the atherosclerosis progression and calcification especially in the scope of the LMCA. Routine control coronary angiography seems to be appropriate to detect important LMCA narrowing, which might improve the long-term prognosis.

\section{References}

1. Naik H, White AJ, Chakravarty T, et al. A meta-analysis of 3,773 patients treated with percutaneous coronary intervention or surgery for unprotected left main coronary artery stenosis. JACC Cardiovasc Interv 2009; 2: 739-747.

2. Gziut A, Gil R, Kulawik T. Comparative analysis of conservative, percutaneous, and surgical treatment outcomes in patients with significant stenosis of the left main coronary artery during five-year follow-up. Kardiol Pol 2010; 68: 381-390.

3. Wijns W, Kolh P, Danchin, et al. Guidelines on myocardial revascularization. Task Force on Myocardial Revascularization of the European Society of Cardiology (ESC) and the European Association for Cardio-Thoracic Surgery (EACTS); European Association for Percutaneous Cardiovascular Interventions (EAPCI). Eur Heart J 2010; 31: 2501-2555.

4. Apostolidou E, Kalisetti D, Logani S, et al. Myocardial revascularization in patients with left main coronary disease. J Invasive Cardiol 2013; 25: 201-217.

5. Ertas G, Ural E, Van der Giessen WJ. Successful stenting of catheter-induced unprotected left main coronary artery dissection. Cardiovasc J Afr 2012; 23: e6-e7.

6. Lee SW, Hong MK, Kim YH, et al. Bail-out stenting for left main coronary artery dissection during catheter-based procedure: acute and long-term results. Clin Cardiol 2004; 27: 393-395.

7. Akgul F, Batyraliev T, Besnili F, et al. Emergency stenting of unprotected left main coronary artery after acute catheter-induced occlusive dissection. Tex Heart Inst J 2006; 33: 515-518. 
8. Al-Saif SM, Liu MW, Al-Mubarak N, et al. Percutaneous treatment of catheter-induced dissection of the left main coronary artery and adjacent aortic wall: a case report. Catheter Cardiovasc Interv 2000; 49: 86-89.

9. Cines DB, Pollak ES, Busk CA, et al. Endothelial cells in physiology and in the pathophysiology of vascular disorders. Blood 1998; 91: 3527-3561.

10. Kuliczkowski W, Kobusiak-Prokopowicz M, Jołda-Mydłowsska B. The endothelial dysfunction in patients with coronary and carotid arteries atherosclerosis. Adv Clin Exp Med 2004; 13: 41-47. 